\title{
Correction to: Secretomes reveal several novel proteins as well as TGF- $\beta 1$ as the top upstream regulator of metastatic process in breast cancer
}

\author{
Nuray $\operatorname{Erin}^{1}{ }^{1} \cdot$ Nur Ogan $^{1} \cdot$ Azmi Yerlikaya $^{2}$ \\ Published online: 2 May 2018 \\ ๑) Springer Science+Business Media, LLC, part of Springer Nature 2018
}

\section{Correction to: Breast Cancer Research and Treatment https://doi.org/10.1007/s10549-018-4752-8}

In the original publication of the article, Acknowledgement section was missed out and Table 1 was published incompletely. The Acknowledgment and complete Table 1 are given in this correction. The original article has been corrected.

The original article can be found online at https://doi.org/10.1007/ s10549-018-4752-8.

Nuray Erin

nerin@akdeniz.edu.tr

1 Department of Medical Pharmacology, School of Medicine, Akdeniz University, B-blok kat 1, SBAUM/Immunoloji Lab, Antalya, Turkey

2 Department of Medical Biology, School of Medicine, Dumlupınar University, Kütahya, Turkey 
Table 1 Proteins secreted into the conditioned medium by highly metastatic breast cancer cells 4TBM, 4TLM and 4THM as compared to the non-metastatic $67 \mathrm{NR}$ breast cancer cell line

\begin{tabular}{|c|c|c|c|c|c|c|c|c|}
\hline \multirow[t]{2}{*}{ No } & \multirow[t]{2}{*}{ Protein name } & \multirow[t]{2}{*}{ Symbol } & \multirow[t]{2}{*}{ Protein access no } & \multirow[t]{2}{*}{ Peptide sequence detected } & \multirow[t]{2}{*}{ Anova $p$-value } & \multicolumn{3}{|c|}{ Average fold change } \\
\hline & & & & & & 4TBM & 4TLM & 4THM \\
\hline \multirow[t]{3}{*}{1} & \multirow[t]{3}{*}{ Collagen, type V, alpha 1} & \multirow[t]{3}{*}{ COL5A1 } & \multirow[t]{3}{*}{ O88207 } & FGFEVGPACFLG & 0.0062 & 8.2 & 1.3 & 7.7 \\
\hline & & & & SSSEPDVAYR & 0.0469 & 15.6 & 2.8 & 11.3 \\
\hline & & & & $\begin{array}{l}\text { DLQLCHPDFPDGEYWVDP- } \\
\text { NQGCSR }\end{array}$ & 0.0021 & 7.1 & 0.93 & 6.4 \\
\hline \multirow[t]{3}{*}{2} & \multirow[t]{3}{*}{ Complement component 3} & \multirow[t]{3}{*}{ C3 } & \multirow[t]{3}{*}{ P01027 } & LITQGENCIK & 0.0072 & 3.4 & 38.3 & 20.1 \\
\hline & & & & DTWVEHWPEAEECQDQK & 0.0034 & 1.6 & 19.9 & 12 \\
\hline & & & & QIFSAEFEVK & 0.0043 & 5.4 & 45.1 & 28.3 \\
\hline \multirow[t]{7}{*}{3} & \multirow[t]{7}{*}{ Laminin, beta 1} & \multirow[t]{7}{*}{ LAMB1 } & \multirow[t]{7}{*}{ P02469 } & AELLLEEAK & 0.0117 & 14.6 & 17.0 & 12.1 \\
\hline & & & & NSDIQGALDSITK & 0.0018 & 7.1 & 11.3 & 6.9 \\
\hline & & & & EGFYDLSAEDPYGCK & 0.0032 & 7.8 & 7.0 & 5.6 \\
\hline & & & & QNAQDVLLK & 0.0141 & 5.8 & 8.9 & 5.0 \\
\hline & & & & LHTLGDNLLDSR & 0.005 & 11.5 & 8.0 & 8.4 \\
\hline & & & & CVCNYLGTVK & 0.0093 & 5.9 & 7.8 & 4.3 \\
\hline & & & & AAQNSGEAEYIEK & 0.0074 & 9.2 & 10.6 & 8.3 \\
\hline \multirow[t]{2}{*}{4} & \multirow{2}{*}{$\begin{array}{l}\text { Transforming growth factor, } \\
\text { beta } 1\end{array}$} & \multirow[t]{2}{*}{ TGFB1 } & \multirow[t]{2}{*}{ P04202 } & VAGESADPEPEPEADYYAK & 0.0055 & 23.4 & 30.6 & 41.3 \\
\hline & & & & QWLNQGDGIQGFR & 0.0021 & 10.5 & 19.0 & 20.4 \\
\hline 5 & Heat shock protein $90 \mathrm{kDa}$ beta & HSP90B1 & P08113 & DISTNYYASQK & 0.0152 & 15.8 & 14.4 & 10.0 \\
\hline & (Grp94), member 1 & & & GLFDEYGSK & 0.0134 & 11.6 & 9.8 & 5.2 \\
\hline & & & & EAESSPFVER & 0.0077 & 12.7 & 11.2 & 7.6 \\
\hline & & & & FAFQAEVNR & 0.0115 & 7.9 & 7.2 & 4.4 \\
\hline & & & & SILFVPTSAPR & 0.0044 & 14.5 & 13.9 & 8.0 \\
\hline & & & & EESDDEAAVEEEEEEK & 0.0104 & 43.6 & 39.2 & 26.9 \\
\hline & & & & TETVEEPLEEDEAAK & 0.0094 & 12.4 & 12.5 & 7.7 \\
\hline & & & & TFEINPR & 0.0047 & 13.4 & 13.8 & 8.3 \\
\hline & & & & YLNFVK & 0.0131 & 12.5 & 13.8 & 7.7 \\
\hline 6 & Fibronectin 1 & FN1 & P11276 & ISCTIANR & 0.0113 & 2.9 & 22.6 & 12.7 \\
\hline & & & & IGDQWDK & 0.0309 & 0.55 & 4.7 & 2.3 \\
\hline & & & & DSMIWDCTCIGAGR & 0.022 & 0.79 & 10.2 & 3.6 \\
\hline & & & & TYLGNALVCTCYGGSR & 0.0381 & 1.1 & 10.6 & 4.1 \\
\hline & & & & YSFCTDHAVLVQTR & 0.026 & 0.82 & 10.8 & 3.7 \\
\hline & & & & GVTYNIIVEALQNQR & 0.0287 & 1.2 & 9.7 & 4.5 \\
\hline & & & & WCGTTQNYDADQK & 0.0396 & 0.6 & 6.5 & 2.5 \\
\hline & & & & GLAFTDVDVDSIK & 0.0326 & 1.2 & 6.9 & 3.4 \\
\hline & & & & $\begin{array}{l}\text { TASPDQTEMTIEGLQPT- } \\
\text { VEYVVSVYAQNR }\end{array}$ & 0.0224 & 0.8 & 6.7 & 3.8 \\
\hline & & & & LTCQCLGFGSGHFR & 0.0498 & 0.8 & 11.0 & 3.9 \\
\hline & & & & $\begin{array}{l}\text { RPGAAEPSPDGTTGHTYN- } \\
\text { QYTQR }\end{array}$ & 0.0456 & 0.79 & 6.8 & 3.5 \\
\hline & & & & TYHVGEQWQK & 0.0161 & 0.71 & 7.8 & 2.9 \\
\hline & & & & IAWESPQGQVSR & 0.0324 & 0.93 & 6.0 & 2.8 \\
\hline & & & & HYQINQQWER & 0.0181 & 0.72 & 6.8 & 2.4 \\
\hline & & & & CFDHAAGTSYVVGETWEK & 0.0375 & 0.91 & 8.7 & 3.5 \\
\hline & & & & PVSINYK & 0.018 & 0.80 & 11.5 & 3.9 \\
\hline & & & & $\begin{array}{l}\text { TEIDKPSQMQVTDVQDN- } \\
\text { SISVR }\end{array}$ & 0.0423 & 3.8 & 41.7 & 17.7 \\
\hline
\end{tabular}


Table 1 (continued)

\begin{tabular}{|c|c|c|c|c|c|c|c|c|}
\hline \multirow[t]{2}{*}{ No } & \multirow[t]{2}{*}{ Protein name } & \multirow[t]{2}{*}{ Symbol } & \multirow[t]{2}{*}{ Protein access no } & \multirow[t]{2}{*}{ Peptide sequence detected } & \multirow[t]{2}{*}{ Anova $p$-value } & \multicolumn{3}{|c|}{ Average fold change } \\
\hline & & & & & & 4TBM & 4TLM & 4THM \\
\hline \multirow[t]{6}{*}{7} & \multirow{6}{*}{$\begin{array}{l}\text { Heat shock } 706 \mathrm{kDa} \text { protein } 5 \\
\text { \{glucose- regulated protein, } \\
78 \mathrm{kDa}\}\end{array}$} & \multirow[t]{6}{*}{ HSPA5 } & \multirow[t]{6}{*}{ P20029 } & ETAEAYLGK & 0.0004 & 6.9 & 8.1 & 8.4 \\
\hline & & & & VYEGERPLTK & 0.0011 & 25.6 & 27.4 & 24.6 \\
\hline & & & & ITITNDQNR & 0.0005 & 48.8 & 38.7 & 76.1 \\
\hline & & & & IEWLESHQDADIEDFK & 0.0009 & 38.9 & 32.7 & 32.5 \\
\hline & & & & SQIFSTASDNQPTVTIK & 0.0023 & 18.1 & 18.0 & 15.5 \\
\hline & & & & NQLTSNPENTVFDAK & 0.0012 & 8.7 & 8.9 & 12.2 \\
\hline \multirow[t]{4}{*}{8} & \multirow{4}{*}{$\begin{array}{l}\text { Milk fat globule-EGF factor } 8 \\
\text { protein }\end{array}$} & \multirow[t]{4}{*}{ MFGE8 } & \multirow[t]{4}{*}{ P21956 } & QVTGIITQGAR & 0.018 & 2.4 & 1.2 & 2.0 \\
\hline & & & & EWLQVDLGTQR & 0.0242 & 2.4 & 1.4 & 2.3 \\
\hline & & & & $\begin{array}{l}\text { VAHSDDGVQWTVY- } \\
\text { EEQGSSK }\end{array}$ & 0.0264 & 3.0 & 1.5 & 2.9 \\
\hline & & & & WGPELAR & 0.0168 & 2.1 & 1.1 & 1.9 \\
\hline \multirow[t]{5}{*}{9} & \multirow{5}{*}{$\begin{array}{l}\text { Peptidylprolyl isomerase B } \\
\text { (cyclophilin B.) }\end{array}$} & \multirow[t]{5}{*}{ PPIB } & \multirow[t]{5}{*}{ P24369 } & TVDNFVALATGEK & 0.003 & 5.3 & 9.6 & 5.4 \\
\hline & & & & VVFGLFGK & 0.0178 & 18.0 & 26.5 & 15.8 \\
\hline & & & & DTNGSQFFITTVK & 0.0008 & 7.2 & 15.2 & 8.2 \\
\hline & & & & DFMIQGGDFTR & 0.0293 & 19.8 & 29.1 & 18.3 \\
\hline & & & & VYFDLQIGDESVGR & 0.035 & 11.3 & 14.3 & 9.8 \\
\hline \multirow[t]{8}{*}{10} & \multirow{8}{*}{$\begin{array}{l}\text { Protein disulfide-isomerase } \\
\text { family A, member } 3\end{array}$} & \multirow[t]{8}{*}{ PDIA3 } & P27773 & TADGIVSHLK & 0.0271 & 9.4 & 9.2 & 8.1 \\
\hline & & & & DASVVGFFR & 0.0241 & 48.4 & 45.3 & 22.6 \\
\hline & & & & YGVSGYPTLK & 0.0212 & 5.4 & 6.1 & 4.4 \\
\hline & & & & SEPIPESNEGPVK & 0.0049 & 9.0 & 9.1 & 6.7 \\
\hline & & & & FAHTNIESLVK & 0.0043 & 8.6 & 9.5 & 7.3 \\
\hline & & & & $\begin{array}{l}\text { TFSHELSDFGLESTT- } \\
\text { GEVPVVAIR }\end{array}$ & 0.0044 & 7.1 & 6.9 & 5.6 \\
\hline & & & & VVVAENFDDIVNEEDK & 0.0024 & 10.1 & 10.0 & 9.3 \\
\hline & & & & EATNPPIIQEEK & 0.005 & 9.5 & 9.8 & 8.0 \\
\hline 11 & Granulin & GRN & P28798 & $\begin{array}{l}\text { LNSGDWGCCPIPEAVCCS- } \\
\text { DNQHCCPQGFTCLAQGY- } \\
\text { CQK }\end{array}$ & 0.0483 & 58.3 & 5.6 & 23.8 \\
\hline & & & & $\begin{array}{l}\text { QTTPLQIG- } \\
\text { DIGCDQHTSCPVGQTC- } \\
\text { CPSLK }\end{array}$ & 0.0263 & 24.5 & 4.9 & 10.2 \\
\hline 12 & Matrix metallopeptidase 3 & MMP3 & P28862 & CGVPDVGGFSTFPGSPK & 0.0006 & 9.3 & 25.4 & 8.7 \\
\hline & & & & YLENYYGLAK & 0.0007 & 17.8 & 68.0 & 21.0 \\
\hline & & & & TYFFVEDK & $<0.0001$ & 19.0 & 65.9 & 21.3 \\
\hline 13 & Thrombospondin 1 & THBS1 & P35441 & IMADSGPIYDK & 0.0379 & 7.4 & 18.0 & 10.7 \\
\hline & & & & GDVNDNFQGVLQNVR & 0.02 & 11.7 & 25.8 & 21.5 \\
\hline & & & & DNCPNLPNSGQEDYDK & 0.0361 & 10.3 & 19.4 & 18.4 \\
\hline & & & & AQGYSGLSVK & 0.042 & 6.2 & 15.7 & 11.1 \\
\hline 14 & Protein tyrosine phosphatase, & PTPRK & P35822 & IFLNWK & 0.0113 & 25.4 & 7.4 & 19.5 \\
\hline & & & & $\begin{array}{l}\text { VLLTRPGEGGTGLPGP- } \\
\text { PLITR }\end{array}$ & 0.0118 & 8.0 & 4.4 & 7.1 \\
\hline 15 & Matrix metallopeptidase 9 & MMP9 & P41245 & VSFQNEVNK & 0.0112 & 0.30 & 2.3 & 2.5 \\
\hline & & & & ALLFSK & 0.0018 & 0.22 & 3.6 & 2.9 . \\
\hline & & & & LGLGPEVTHVSGLLPR & 0.012 & 0.12 & 3.2 & 2.9 \\
\hline & & & & QLSLPQTGELDSQTLK & 0.0079 & 0.33 & 2.4 & 2.6 \\
\hline & & & & GSPLQGPFLTAR & 0.0062 & 0.35 & 2.4 & 2.6 \\
\hline & & & & WCATTANYDQDK & 0.0032 & 0.13 & 2.1 & 2.6 \\
\hline & & & & QPTFVVFPK & 0.0159 & 0.29 & 2.9 & 2.9 \\
\hline & & & & AFAVWGEVAPLTFTR & 0.0018 & 0.23 & 2.0 & 2.4 \\
\hline & & & & TWPALPATLDSAFEDPQTK & 0.0021 & 0.28 & 2.2 & 3.1 \\
\hline
\end{tabular}


Table 1 (continued)

\begin{tabular}{|c|c|c|c|c|c|c|c|c|}
\hline \multirow[t]{2}{*}{ No } & \multirow[t]{2}{*}{ Protein name } & \multirow[t]{2}{*}{ Symbol } & \multirow[t]{2}{*}{ Protein access no } & \multirow[t]{2}{*}{ Peptide sequence detected } & \multirow[t]{2}{*}{ Anova $p$-value } & \multicolumn{3}{|c|}{ Average fold change } \\
\hline & & & & & & 4TBM & 4TLM & 4THM \\
\hline \multirow[t]{10}{*}{16} & \multirow[t]{10}{*}{ Bone morphogenetic protein 1} & \multirow[t]{10}{*}{ BMP1 } & \multirow[t]{10}{*}{ P98063 } & LPEPIVSTDSR & 0.0001 & 12.3 & 12.6 & 11.1 \\
\hline & & & & GFQASHSTECGGQVR & $<0.0001$ & 18.1 & 15.6 & 17.3 \\
\hline & & & & VTSTSGTITSPNWPDK & 0.0001 & 76.9 & 87.2 & 71.6 \\
\hline & & & & ENIQPGQEYNFLK & 0.0001 & 24.6 & 21.2 & 24.0 \\
\hline & & & & FVSDGSINK & $<0.0001$ & 21.7 & 22.8 & 20.3 \\
\hline & & & & FYSDNSVQR & 0.0002 & 26.8 & 23.4 & 21.9 \\
\hline & & & & CSCDPGYELAPDK & 0.002 & 8.4 & 8.6 & 6.9 \\
\hline & & & & YDFVEVR & $<0.0001$ & 61.4 & 57.3 & 54.9 \\
\hline & & & & NCIWQLVAPTQYR & $<0.0001$ & 34.8 & 33.8 & 31.7 \\
\hline & & & & GGGPQAISIGK & 0.0002 & 29.6 & 22.9 & 23.0 \\
\hline \multirow[t]{7}{*}{17} & \multirow[t]{7}{*}{ Carboxypeptidase E } & \multirow[t]{7}{*}{$\mathrm{CPE}$} & \multirow[t]{7}{*}{ Q00493 } & IHIMPSLNPDGFEK & 0.0133 & 3.7 & 10.2 & 5.5 \\
\hline & & & & SNAQGIDLNR & 0.0311 & 4.1 & 7.7 & 5.3 \\
\hline & & & & LTASAPGYLAITK & 0.0243 & 3.6 & 9.8 & 5.2 \\
\hline & & & & LLAPGNYK & 0.036 & 3.3 & 7.8 & 3.8 \\
\hline & & & & $\begin{array}{l}\text { SGTAHEYSSCPD- } \\
\text { DAIFQSLAR }\end{array}$ & 0.0266 & 3.1 & 5.4 & 3.8 \\
\hline & & & & DGDYWR & 0.0456 & 6.0 & 13.6 & 8.7 \\
\hline & & & & EALVSVWLQCTAISR & 0.0241 & 4.5 & 7.7 & 6.6 \\
\hline \multirow[t]{3}{*}{18} & \multirow[t]{3}{*}{ Heparan sulfate proteoglycan 2} & \multirow[t]{3}{*}{ HSPG2 } & \multirow[t]{3}{*}{ Q05793 } & DIIYIGGAPDVATLTR & 0.0079 & 13.1 & 8.3 & 10.5 \\
\hline & & & & TSTADGLLLWQGVVR & 0.0268 & 6.4 & 3.3 & 5.5 \\
\hline & & & & CLCLPGFSGPR & 0.0489 & 4.0 & 2.1 & 3.0 \\
\hline \multirow[t]{2}{*}{19} & \multirow{2}{*}{$\begin{array}{l}\text { Amyloid beta (A4) precursor- } \\
\text { like protein } 2\end{array}$} & \multirow[t]{2}{*}{ APLP2 } & \multirow[t]{2}{*}{ Q06335 } & LNMHVNIQTGK & 0.0123 & 40.5 & 10.6 & 30.8 \\
\hline & & & & QQLVETHLAR & 0.0003 & 5.0 & 14.5 & 9.2 \\
\hline \multirow[t]{2}{*}{20} & \multirow{2}{*}{$\begin{array}{l}\text { Serpin peptidase inhibitor, } \\
\text { clade } E \text { (nexin, plasminogen } \\
\text { activator inhibitor type } 1 \text { ) }\end{array}$} & \multirow[t]{2}{*}{ SERPINE2 } & Q07235 & GMIDNLLSPNLIDGALTR & 0.0137 & 7.9 & 9.8 & 16.5 \\
\hline & & & & $\begin{array}{l}\text { SRPHENVVVSPHGIASIL- } \\
\text { GMLQLGADGK }\end{array}$ & 0.0412 & 6.4 & 7.6 & 11.0 \\
\hline 21 & Fibulin 1 & FBLN1 & Q08879 & TCVDINECQR & 0.0398 & 5.6 & 3.1 & 3.2 \\
\hline & & & & CVDVDECSPPAEPCGK & 0.0178 & 56.4 & 32.2 & 25.5 \\
\hline & & & & $\begin{array}{l}\text { DIDECETGIHNCPPDFIC- } \\
\text { QNTLGSFR }\end{array}$ & 0.0007 & 13.4 & 7.7 & 7.0 \\
\hline & & & & AGFYFDGISR & 0.0015 & 9.4 & 7.5 & 5.9 \\
\hline & & & & CLSFECPENYRR & 0.0405 & 3.7 & 2.9 & 2.1 \\
\hline & & & & $\begin{array}{l}\text { ARENSDFVQGNGADLQD- } \\
\text { PAK }\end{array}$ & 0.0011 & 4.1 & 2.9 & 2.4 \\
\hline & & & & DSSCGTGYELTEDNNCK & 0.0011 & 17.8 & 8.2 & 8.7 \\
\hline 22 & Laminin, alpha 5 & LAMAS & Q61001 & $\begin{array}{l}\text { APDQPLDSPHVCRPCDCES- } \\
\text { DFTDGTCEDLTGR }\end{array}$ & 0.014 & 22.1 & 24.6 & 21.7 \\
\hline & & & & STDFGHTYQPWQFFASSK & 0.0432 & 8.8 & 13.1 & 9.5 \\
\hline & & & & FANSPRPDLWVLER & 0.0197 & 6.9 & 12.1 & 7.8 \\
\hline & & & & AHPVSNAIDGTER & 0.0007 & 17.6 & 28.7 & 20.9 \\
\hline
\end{tabular}


Table 1 (continued)

\begin{tabular}{|c|c|c|c|c|c|c|c|c|}
\hline \multirow[t]{2}{*}{ No } & \multirow[t]{2}{*}{ Protein name } & \multirow[t]{2}{*}{ Symbol } & \multirow[t]{2}{*}{ Protein access no } & \multirow[t]{2}{*}{ Peptide sequence detected } & \multirow[t]{2}{*}{ Anova $p$-value } & \multicolumn{3}{|c|}{ Average fold change } \\
\hline & & & & & & 4TBM & 4TLM & 4THM \\
\hline \multirow[t]{10}{*}{23} & \multirow[t]{10}{*}{ Ceruloplasmin (ferroxidase) } & \multirow[t]{10}{*}{$\mathrm{CP}$} & \multirow[t]{10}{*}{ Q61147 } & MYYSGVDPTK & 0.0478 & 35.5 & 23.3 & 27.5 \\
\hline & & & & TYTWQIPER & 0.0327 & 17.4 & 7.4 & 10.7 \\
\hline & & & & SLTLLMNPDTK & 0.0218 & 49.5 & 20.0 & 30.0 \\
\hline & & & & ALYFEYTDGTFSK & 0.0083 & 47.2 & 17.6 & 29.6 \\
\hline & & & & DIFTGLIGPMK & 0.0493 & 11.0 & 4.0 & 7.1 \\
\hline & & & & DIASGLIGPLILCK & 0.0097 & 69.6 & 25.8 & 39.6 \\
\hline & & & & $\begin{array}{l}\text { VLPGQQYVYVLHANEP- } \\
\text { SPGEGDSNCVTR }\end{array}$ & 0.0161 & 17.1 & 7.9 & 11.4 \\
\hline & & & & VFFEQGATR & 0.0036 & 105.6 & 26.7 & 58.5 \\
\hline & & & & EYTDGSFTNRK & 0.0109 & 65.0 & 25.6 & 43.4 \\
\hline & & & & $\begin{array}{l}\text { GPDEEHLGILGPVIWAEVG- } \\
\text { DTIK }\end{array}$ & 0.0191 & 18.8 & 3.8 & 10.5 \\
\hline \multirow[t]{5}{*}{24} & \multirow{5}{*}{$\begin{array}{l}\text { Insulin-like growth factor bind- } \\
\text { ing protein } 7\end{array}$} & \multirow{5}{*}{ IGFBP7 } & \multirow[t]{5}{*}{ Q61581 } & DACGCCPVCAR & 0.0273 & 0.84 & 8.8 & 13.4 \\
\hline & & & & VFLSCEVIGIPTPVWNK & 0.0291 & 0.79 & 9.6 & 13.9 \\
\hline & & & & ITVVDALHEIPLK & 0.0484 & 1.0 & 10.1 & 13.5 \\
\hline & & & & TELLPGDRENLAIQTR & 0.0414 & 0.68 & 10.7 & 13.2 \\
\hline & & & & $\begin{array}{l}\text { EDAGEYECHASN- } \\
\text { SQGQASAAAK }\end{array}$ & 0.0405 & 0.61 & 8.5 & 10.9 \\
\hline \multirow[t]{2}{*}{25} & \multirow[t]{2}{*}{ Glucosidase, alpha: neutral AB } & \multirow[t]{2}{*}{ GANAB } & \multirow[t]{2}{*}{ Q88HN3 } & NPEPELLVR & 0.015 & 8.6 & 8.4 & 10.7 \\
\hline & & & & $\begin{array}{l}\text { DPAEGNGAQPEATPGDGD- } \\
\text { KPEETQEK }\end{array}$ & 0.0201 & 10.2 & 5.5 & 13.3 \\
\hline \multirow[t]{7}{*}{26} & \multirow{7}{*}{$\begin{array}{l}\text { Quiescin Q6 sulfhydryl oxi- } \\
\text { dase } 1\end{array}$} & \multirow[t]{7}{*}{ QSOX1 } & Q8BND5 & EFNIAGFPTVR & 0.0111 & 50.7 & 37.3 & 40.7 \\
\hline & & & & VLNTESDLVNK & 0.0044 & 26.0 & 22.1 & 20.6 \\
\hline & & & & LSGALSEDPHFPK & 0.0151 & 13.4 & 14.9 & 9.7 \\
\hline & & & & AHFSPANIVIDSSASR & 0.0008 & 30.8 & 28.8 & 24.5 \\
\hline & & & & SFYTSYLR & 0.0008 & 58.8 & 50.1 & 47.9 \\
\hline & & & & FFQAFTK & 0.0014 & 19.8 & 17.7 & 15.0 \\
\hline & & & & $\begin{array}{l}\text { ELCSACHNELNGQVPL- } \\
\text { WDLGATLNFLK }\end{array}$ & 0.0106 & 26.2 & 15.6 & 22.3 \\
\hline 27 & $\begin{array}{l}\text { Latent transforming growth } \\
\text { factor beta binding protein } 1\end{array}$ & LTBP1 & Q8CG19 & $\begin{array}{l}\text { FLNSFEELQAEECGILNG- } \\
\text { CENGR }\end{array}$ & 0.0114 & 11.4 & 5.0 & 7.2 \\
\hline & & & & EAQPGQSQVSYQGLPVQK & 0.049 & 4.2 & 4.1 & 3.9 \\
\hline 28 & Latent transforming growth & LTBP4 & Q8K4G1 & GGYTCVCPDGFLLDSSR & 0.0366 & 4.7 & 4.6 & 4.7 \\
\hline & factor beta binding protein 4 & & & CVPRPSGYTCACDPGFR & 0.0451 & 4.3 & 6.2 & 4.8 \\
\hline & & & & GSFPEPEESSER & 0.0037 & 58.0 & 21.2 & 27.7 \\
\hline & & & & CVCPAGFR & 0.0138 & 5.8 & 6.6 & 6.2 \\
\hline & & & & $\begin{array}{l}\text { GSYTGALSEPYEGLEAEEC- } \\
\text { GILDGCPHGR }\end{array}$ & 0.0005 & 31.4 & 17.1 & 17.3 \\
\hline 29 & OAF homolog (Drosophila) & OAF & Q8CQZR4 & ALEQAELPR & 0.0062 & 2.5 & 6.4 & 4.5 \\
\hline & & & & GQSQFQALCFVTR & 0.0166 & 2.3 & 5.2 & 4.2 \\
\hline & & & & SYTFDFYVPQK & 0.0068 & 2.8 & 53 & 4.9 \\
\hline & & & & ALILGELEK & 0.0024 & 2.6 & 7.0 & 5.2 \\
\hline & & & & GVDSSVFEALPK & 0.0024 & 3.0 & 6.5 & 5.7 \\
\hline 30 & Protein disulfide isomerase & PDIA6 & Q922R8 & GSFSEQGINEFLR & 0.0119 & 25.9 & 16.2 & 12.6 \\
\hline & family A, member 6 & & & NLEPEWAAAATEVK & 0.0381 & 14.1 & 12.1 & 7.5 \\
\hline 31 & Tubulointerstitial nephritis & TINAGL1 & Q99JR5 & ITGWGEETLPDGR & $<0.0001$ & 25.6 & 6.9 & 30.0 \\
\hline & antigen-like 1 & & & IYPVFGTYWDNCNR & 0.0014 & 11.9 & 2.8 & 11.8 \\
\hline & & & & YWTAANSWGPWWGER & 0.0003 & 19 & 1.8 & 5.1 \\
\hline 32 & Thymic stromal lymphopoietin & TSLP & Q9JIE6 & FEQIEDCESK & 0.011 & 58.4 & 25.3 & 29.3 \\
\hline & & & & IYCNIIFHDLTGDLK & 0.0281 & 51.9 & 24.9 & 25.8 \\
\hline
\end{tabular}


Table 1 (continued)

\begin{tabular}{|c|c|c|c|c|c|c|c|c|}
\hline \multirow[t]{2}{*}{ No } & \multirow[t]{2}{*}{ Protein name } & \multirow[t]{2}{*}{ Symbol } & \multirow[t]{2}{*}{ Protein access no } & \multirow[t]{2}{*}{ Peptide sequence detected } & \multirow[t]{2}{*}{ Anova $p$-value } & \multicolumn{3}{|c|}{ Average fold change } \\
\hline & & & & & & 4TBM & 4TLM & 4THМ \\
\hline \multirow[t]{2}{*}{33} & \multirow{2}{*}{$\begin{array}{l}\text { Procollagen-lysine, 2-oxogluta- } \\
\text { rate 5-dioxygenase } 1\end{array}$} & \multirow[t]{2}{*}{ PLOD1 } & \multirow[t]{2}{*}{ Q9ROE2 } & SEDYVDIVQGR & 0.0132 & 6.0 & 0.60 & 2.7 \\
\hline & & & & VGVWNVPYISNIYLIK & 0.0306 & 8.5 & 0.23 & 2.5 \\
\hline \multirow[t]{4}{*}{34} & \multirow[t]{4}{*}{ Cathepsin Z } & \multirow[t]{4}{*}{ CTSZ } & \multirow[t]{4}{*}{ Q9WUU7 } & NSWGEPWGEK & 0.0138 & 7.6 & 6.7 & 5.0 \\
\hline & & & & FNQCGTCTEFK & 0.0208 & 7.0 & 6.0 & 53 \\
\hline & & & & VGDYGSLSGR & 0.0178 & 10.0 & 6.4 & 6.6 \\
\hline & & & & HGIPDETCNNYQAK & 0.01 & 11.2 & 10.1 & 8.0 \\
\hline \multirow[t]{3}{*}{35} & \multirow{3}{*}{$\begin{array}{l}\text { EGF containing fibulin-like } \\
\text { extracellular matrix protein } 2\end{array}$} & \multirow[t]{3}{*}{ EFEMP2 } & \multirow[t]{3}{*}{ Q9WVJ9 } & SCVDVNECDMGAPCEQR & 0.0054 & 44.6 & 5.6 & 29.7 \\
\hline & & & & FSCHCPQGYQLLATR & 0.0007 & 29.9 & 93 & 21.8 \\
\hline & & & & $\begin{array}{l}\text { SVPADVFQIQATSVYP- } \\
\text { GAYNAFQIR }\end{array}$ & 0.0101 & 26.0 & 7.1 & 18.9 \\
\hline
\end{tabular}

Proteins were detected by label-free nLC-MS/MS. Proteins with at least 2 or more peptides detected were presented. The statistical significance was determined by one-way ANOVA at $p<0.05(n=3)$

Acknowledgements This study was supported by TUBI-

TAK. Grant No: $115 Z 286$. 